\title{
Ischemic Stroke and Septic ShockAfter Subacute Endocarditis Caused by Haemophilus parainfluenzae: Case Report
}

\author{
Mayra Goncalves Meneguetia Jaciara Machado-Viana ${ }^{\mathrm{b}}$, Gilberto Gambero Gaspara, \\ Edson Antonio Nicolini ${ }^{\mathrm{b}}$, Anibal Basile-Filho ${ }^{\mathrm{b}}$, Maria Auxiliadora-Martins ${ }^{\mathrm{b}, \mathrm{c}}$
}

\begin{abstract}
Haemophilus parainfluenzae, which belongs to the HACEK (Haemophilus ssp, Actinobacillus actinomycetemcomitans, Cardiobacterium hominis, Eikenella corrodens, and Kingella kingae) group, is a rare cause of subacute endocarditis and may lead to ischemic stroke. A 65-year-old female patient previously diagnosed with rheumatic valve disease was submitted to surgical mitral valve repair in 1996. Physical examination did not reveal any murmurs; physical examination of the lungs and abdomen was normal. The patient was admitted to hospital with progressive dyspnea, dry cough, and fever. Transesophageal echocardiogram revealed an approximately $8-\mathrm{mm}$ filamentous image with chaotic motion in the ventricular face of the anterior mitral valve leaflet compatible with vegetation. Treatment with ceftriaxone and gentamicin was initiated. Haemophilus parainfluenzae grew in five blood culture samples. Along the hospital stay, the patient's level of consciousness decreased, and she was diagnosed with ischemic stroke of cardioembolic etiology. The patient developed septic shock refractory to the prescribed treatment and died 12 days after admission. Even though the patient started being treated for endocarditis before the infectious agent was identified, the prompt use of antimicrobials hindered the growth of Haemophilus parainfluenzae and made its isolation difficult.
\end{abstract}

Keywords: Haemophilus parainfluenzae; Infective endocarditis; Septic shock; Stroke

\section{Introduction}

Infective endocarditis is a disease that affects the endocardium

Manuscript accepted for publication August 18, 2016

${ }^{a}$ Committee for Hospital Infection Control, Department of Medical Clinics, University Hospital, Ribeirao Preto Medical School, University of Sao Paulo, Ribeirao Preto, SP, Brazil

bIntensive Therapy Division, Department of Surgery and Anatomy, University Hospital, Ribeirao Preto Medical School, University of Sao Paulo, Ribeirao Preto, SP, Brazil

${ }^{c}$ Corresponding Author: Maria Auxiliadora-Martins, Divisao de Terapia Intensiva, Departamento de Cirurgia e Anatomia, Hospital das Clinicas da Faculdade de Medicina de Ribeirao Preto, USP, 2o andar, Av. Bandeirantes, 3900 Bairro Monte Alegre, CEP 14049-900, Ribeirao Preto, SP, Brazil.

Email:mam_martins@hotmail.com

doi: https://doi.org/10.14740/jocmr2703w surface or the vascular endothelium, leading to an inflammatory process that may contain vegetation, fibrin, and microorganisms. Bacteria, viruses, and fungi can cause infective endocarditis; mycobacteria and rickettsia can also lead to endocarditis, albeit less frequently. Aseptic endocarditis may also occur. Infective endocarditis can have acute or subacute progression and affect native or prosthetic valves [1].

The main causative agents of endocarditis are Streptococcus ssp, mainly those belonging to the group viridans, and Staphylococcus ssp. Staphylococcus aureus is generally associated with the use of central venous catheter and injectable drugs. Staphylococcus epidermidis usually infects postoperative cardiac patients. Staphylococcus epidermidis and Enterococcus ssp frequently affect premature infants who use central venous catheter [2].

Bacteria of the HACEK group (Haemophilus ssp, Actinobacillus actinomycetemcomitans, Cardiobacterium hominis, Eikenella corrodens, and Kingella kingae) cause only 5\% of endocarditis cases in adults. Recent studies have revealed an incidence of $0.8-6 \%$ [3-7]. HACEK is a small heterogeneous group of gram-negative bacilli that often colonize the oropharynx. These bacilli have long been recognized as one of the causes of infective endocarditis and are more easily isolated in aerobic media. Their cultures require long incubation time for bacteria to grow [8]. Bacteremia caused by HACEK microorganisms in the absence of an obvious focus of infection highly suggests endocarditis, even in the absence of typical signs [2].

In the past, the HACEK group was sensitive to ampicillin, but B-lactamase-producing strains have emerged. These microorganisms are sensitive to ceftriaxone, other cephalosporins, and third-generation quinolones. Therefore, ceftriaxone has been the treatment of choice for patients with endocarditis due to HACEK [9].

Haemophilus parainfluenzae belongs to the HACEK group. Given the low incidence of this agent, we report a case of subacute infective endocarditis caused by Haemophilus parainfluenzae in a patient with double mitral lesion of rheumatic etiology.

\section{Case Report}

A 65-year-old female patient previously diagnosed with rheumatic valve disease was submitted to surgical mitral valve repair in 1996 and percutaneous exchange of the mitral valve in 2007. This patient visited the cardiology outpatient clinic 
and complained of dyspnea on the slightest effort, paroxysmal nocturnal dyspnea, orthopnea, dry cough, and daily episodes of fever between 38 and $38.5^{\circ} \mathrm{C}$ persisting for 1 month. The patient also reported being treated for dengue fever in another health service 40 days previously. During the hospital stay, the patient experienced an episode of paroxysmal atrial flutter. Ten days later, the patient's level of consciousness decreased abruptly (Glasgow coma scale of 13), and she presented anisocoric pupils. The patient was referred to the intensive care unit (ICU). Upon admission to the ICU, she was drowsy, pale, hydrated, acyanotic, anicteric, and afebrile $\left(37.3^{\circ} \mathrm{C}\right)$. The arterial blood pressure was $122 / 73 \mathrm{~mm} \mathrm{Hg}$, the heart rate was 96 beats per minute, and the respiratory rate was 14 breaths per minute. Heart auscultation was normal; murmurs were absent. Respiratory auscultation and abdomen examination were normal. There was mild edema in the lower limbs.

Laboratorial records revealed the following data: hemoglobin $8.5 \mathrm{~g} / \mathrm{dL}$, hematocrit $25 \%$, white blood cells $16,200 /$ $\mathrm{mm}^{3}$, platelets $192,000 / \mathrm{mm}^{3}$ without left shift and with mild anisocytosis and microcytosis, urea $67 \mathrm{mg} / \mathrm{dL}$, creatinine 2.39 $\mathrm{mg} / \mathrm{dL}$, sodium $132 \mathrm{mmol} / \mathrm{L}$, potassium $3.6 \mathrm{mmol} / \mathrm{L}$, and $\mathrm{C}$ reactive protein $11.2 \mathrm{mg} / \mathrm{dL}$. At admission, the patient's Acute Physiology and Chronic Health Disease Classification System (APACHE) II score was 22 (which corresponded to a death risk of $45 \%)[10,11]$, and her Sequential Organ Failure Assessment (SAPS) III score was 64 (which corresponded to a death risk of 44\%) $[12,13]$. Dental evaluation indicated precarious dentition.

Blood culture samples were collected and empirical treatment with ceftriaxone associated with gentamicin was initiated. Transesophageal echocardiogram revealed an approximately $8-\mathrm{mm}$ filamentous image with chaotic motion in the ventricular face of the anterior mitral valve leaflet compatible with vegetation. After 10 days in hospital, the patient's level of consciousness decreased, and she presented sensitive conduction aphasia and mild right central paralysis. Ischemic stroke with high probability of septic etiology was suspected. Brain tomography demonstrated hypodense left insular lobe corresponding to incipient ischemia. Mechanical thrombectomy was indicated, but the arteriogram did not show occlusion of the proximal vessels. Failed partial filling of the parietal branch of the left middle cerebral artery was observed. The patient progressed with worsened level of consciousness, hemodynamic instability, and need for hemodialysis. Orotracheal intubation was performed, vasoactive drugs were initiated, and brain tomography was repeated. The tomography evidenced brain edema in the posterior circulation region and hypodensity in the left thalamus. The patient presented septic shock refractory to the therapeutic measures and died.

\section{Diagnosis}

The transesophageal echocardiogram evidenced pronounced dilation of the left atrium, moderate double mitral lesion, mild to moderate tricuspid insufficiency, moderate pulmonary hypertension, and approximately 8 - $\mathrm{mm}$ filamentous image with chaotic motion in the ventricular face of the anterior mitral valve leaflet compatible with vegetation.
Haemophilus parainfluenzae grew in the four blood culture samples. Blood culture was conducted in diffusion discs. The microorganism was sensitive to all the tested B-lactamic compounds.

\section{Discussion}

Reporting this clinical case is important because it concerns an uncommon etiological agent and the presence of potentially fatal complications, such as ischemic stroke. The empirical use of antibiotics before the final blood culture results is noteworthy.

Blood culture samples were collected appropriately. The bacterium grew slowly, which is typical of microorganisms of the HACEK group. Accurate microbiological diagnosis is crucial for the correct choice of the antimicrobial agent.

The literature contains few examples of endocarditis caused by Haemophilus parainfluenzae. Due to the growing production of betalactamase by this group of bacteria, thirdgeneration cephalosporins are the treatment of choice [9].

The incidence of systemic embolism is higher when left heart valves are involved and Staphylococcus aureus, Candida, Abiotrophia, or HACEK microorganisms account for the infection. Over $50 \%$ of embolic events involve the central nervous system, whereas $90 \%$ of these events affect the middle cerebral artery $[14,15]$. The main complication regarding involvement of the right chamber is pulmonary embolism, which may progress to abscess formation. Possible severe valve dysfunction or chordae rupture must not be overlooked and may require prompt surgical intervention.

Our patient presented a negative outcome due to complications. This case reinforces the need to insist on the early identification of the etiological agent of endocarditis.

\section{Conclusion}

Apart from the usual causative agents of infectious diseases, infections caused by bacteria of the HACEK group must be suspected in patients with a history of periodontal disease or recent dental treatment.

This study attested to the high sensitivity of Haemophilus parainfluenzae to B-lactamic antimicrobials (penicillin and cephalosporins). Knowing that most empirical regimens to treat endocarditis involve this class of antimicrobial, the patient started being treated even before the etiological agent was isolated. However, early administration of the antimicrobial made isolation of Haemophilus parainfluenzae difficult.

\section{Acknowledgments}

We thank Fundacao de Amparo ao Ensino, Pesquisa e Assistencia (FAEPA, Foundation for the Support of Education, Research and Assistance) of the University Hospital of Ribeirao Preto Medical School, University of Sao Paulo for financial support. 


\section{Conflicts of Interest}

None of the authors have any conflicts of interest to disclose.

\section{Consent}

Written informed consent was obtained from the patient for publication of this case report.

\section{Availability of Data and Materials}

Laboratorial records: Laboratory Hematology of University Hospital, Ribeirao Preto Medical School, University of Sao Paulo, Ribeirao Preto, SP, Brazil.

Blood culture samples: Microbiology Laboratory of University Hospital, Ribeirao Preto Medical School, University of Sao Paulo, Ribeirao Preto, SP, Brazil.

Transesophageal echocardiogram: Cardiology Servisse of University Hospital, Ribeirao Preto Medical School, University of Sao Paulo, Ribeirao Preto, SP, Brazil.

Tomography: Imaging Center of University Hospital, Ribeirao Preto Medical School, University of Sao Paulo, Ribeirao Preto, SP, Brazil.

\section{Author Contributions}

Study design, data analysis and interpretation: MGM, MAM, and GGG. Revising critically for important intellectual content: MGM, MAM, GGG, JMV, EAN, and ABF. All authors have made substantial contributions to the work reported in this manuscript (e.g., data collection, analysis, writing or editing assistance).

\section{Abbreviations}

HACEK: Haemophilus ssp, Actinobacillus actinomycetemcomitans, Cardiobacterium hominis, Eikenella corrodens, and Kingella kingae; ICU: intensive care unit; APACHE II: Acute Physiology and Chronic Health Disease Classification System II; SAPS III: Sequential Organ Failure Assessment III

\section{References}

1. Barbosa MM. [Infective endocarditis: clinical profile in evolution]. Arq Bras Cardiol. 2004;83(3):189-190.

2. Wilson WR, Karchmer AW, Dajani AS, Taubert KA, Bayer A, Kaye D, Bisno AL, et al. Antibiotic treatment of adults with infective endocarditis due to streptococci, enterococci, staphylococci, and HACEK microorganisms. American Heart Association. JAMA. 1995;274(21):17061713.

3. Chambers ST, Murdoch D, Morris A, Holland D, Pappas P,
Almela M, Fernandez-Hidalgo N, et al. HACEK infective endocarditis: characteristics and outcomes from a large, multi-national cohort. PLoS One. 2013;8(5):e63181.

4. Selton-Suty C, Celard M, Le Moing V, Doco-Lecompte T, Chirouze C, Iung B, Strady C, et al. Preeminence of Staphylococcus aureus in infective endocarditis: a 1-year population-based survey. Clin Infect Dis. 2012;54(9):1230-1239.

5. Ferreiros E, Nacinovich F, Casabe JH, Modenesi JC, Swieszkowski S, Cortes C, Hernan CA, et al. Epidemiologic, clinical, and microbiologic profile of infective endocarditis in Argentina: a national survey. The Endocarditis Infecciosa en la Republica Argentina-2 (EIRA-2) Study. Am Heart J. 2006;151(2):545-552.

6. Tleyjeh IM, Steckelberg JM, Murad HS, Anavekar NS, Ghomrawi HM, Mirzoyev Z, Moustafa SE, et al. Temporal trends in infective endocarditis: a populationbased study in Olmsted County, Minnesota. JAMA. 2005;293(24):3022-3028.

7. Wassef N, Rizkalla E, Shaukat N, Sluka M. HACEKinduced endocarditis. BMJ Case Rep. 2013;2013.

8. Berbari EF, Cockerill FR, 3rd, Steckelberg JM. Infective endocarditis due to unusual or fastidious microorganisms. Mayo Clin Proc. 1997;72(6):532-542.

9. Habib G, Lancellotti P, Antunes MJ, Bongiorni MG, Casalta JP, Del Zotti F, Dulgheru R, et al. 2015 ESC Guidelines for the management of infective endocarditis: The Task Force for the Management of Infective Endocarditis of the European Society of Cardiology (ESC). Endorsed by: European Association for Cardio-Thoracic Surgery (EACTS), the European Association of Nuclear Medicine (EANM). Eur Heart J. 2015;36(44):3075-3128.

10. Knaus WA, Draper EA, Wagner DP, Zimmerman JE. APACHE II: a severity of disease classification system. Crit Care Med. 1985;13(10):818-829.

11. Knaus WA, Wagner DP. Multiple systems organ failure: epidemiology and prognosis. Crit Care Clin. 1989;5(2):221-232.

12. Moreno RP, Metnitz PG, Almeida E, Jordan B, Bauer P, Campos RA, Iapichino G, et al. SAPS 3 - From evaluation of the patient to evaluation of the intensive care unit. Part 2: Development of a prognostic model for hospital mortality at ICU admission. Intensive Care Med. 2005;31(10):1345-1355.

13. Metnitz PG, Moreno RP, Almeida E, Jordan B, Bauer P, Campos RA, Iapichino G, et al. SAPS 3 - From evaluation of the patient to evaluation of the intensive care unit. Part 1: Objectives, methods and cohort description. Intensive Care Med. 2005;31(10):1336-1344.

14. Francioli P. Central nervous system complications of infective endocarditis. In: Scheld WM, Whiteley RJ, Durack DT, eds. Infections of the Central Nervous System. New York, NY: Raven Press; 1991:515-559.

15. Heiro M, Nikoskelainen J, Engblom E, Kotilainen E, Marttila R, Kotilainen P. Neurologic manifestations of infective endocarditis: a 17-year experience in a teaching hospital in Finland. Arch Intern Med. 2000;160(18):27812787. 